\title{
Tumor mutational burden (TMB) as a biomarker of response to immunotherapy in small cell lung cancer
}

\author{
Yanis Boumber ${ }^{1,2,3}$ \\ ${ }^{1}$ Program in Molecular Therapeutics, ${ }^{2}$ Department of Hematology/Oncology, Fox Chase Cancer Center, Philadelphia, PA, 19111, USA; Insititute \\ of Fundamental Medicine and Biology, Kazan Federal University, Kazan, Russia \\ Correspondence to: Yanis Boumber, MD, PhD. Assistant Professor, Fox Chase Cancer Center, 333 Cottman Avenue, Philadelphia, PA 19111, USA. \\ Email: Yanis.Boumber@fccc.edu. \\ Provenance: This is an invited Editorial commissioned by the Section Editor Dr. Lei Deng (Department of Medicine, Jacobi Medical Center, Albert \\ Einstein College of Medicine, Bronx, NY, USA). \\ Comment on: Hellmann MD, Callahan MK, Awad MM, et al. Tumor Mutational Burden and Efficacy of Nivolumab Monotherapy and in \\ Combination with Ipilimumab in Small-Cell Lung Cancer. Cancer Cell 2018;33:853-61.e4.
}

Submitted Jul 16, 2018. Accepted for publication Jul 23, 2018.

doi: $10.21037 /$ jtd.2018.07.120

View this article at: http://dx.doi.org/10.21037/jtd.2018.07.120

Tumors avoid immune surveillance by inactivating various T-cell cytotoxic pathways that can promote cell death (1-3). Cytotoxic T lymphocyte antigen 4 (CTLA-4) and programmed death 1 (PD-1) are expressed primarily on cytotoxic T lymphocytes. In contrast, programmed death ligand 1 (PD-L1) is expressed on antigen-presenting lymphoid and non-lymphoid tissues, on tumor cells, and on virus-infected cells. PD-L1 expressed on a target cell engages PD-1, to directly inhibit cytotoxic T-cell action against the target cell (4). Similarly, CTLA-4 engagement by B7-1 (CD80)/B7-2 (CD86), expressed on an antigen presenting cell (APC), provides another key inhibitory checkpoint signal that blocks T-cell activation (1), contributing to tumor survival.

Clinical development of immunotherapy checkpoint inhibitors targeting and disrupting the PD-1/PD-L1 and CTLA-4/B7 interactions has transformed the treatment approaches of solid tumors in recent years, including nonsmall cell lung cancer (NSCLC) $(1,3,5)$. These therapies also show promise in typically more therapy-resistant tumors, such as small cell lung cancer (SCLC). The drugs most thoroughly evaluated in SCLC to date include nivolumab, and pembrolizumab, which target PD-1, and ipilimumab, which targets CTLA-4 protein $(6,7)$. Even though many SCLC patients do not respond to single agent therapy directed against PD-1 or PD-L1, these drug work better when combined with a CTLA-4 inhibitor (8).
Pembrolizumab has been initially approved for NSCLC patients with PD-L1 positive tumors (signal in $\geq 1 \%$ of cells in tumor mass by immunohistochemistry) in second line therapy, based on the Keynote-10 study (9). More recently, approval was granted for patients with high PD-L1 expression (defined as $\geq 50 \%$ of cells being PD-L1 positive) in the first line advanced setting, based on superiority to chemotherapy platinum doublet in a randomized study (10). These two key studies have established PD-L1 as a key biomarker in advanced NSCLC. More recently, tumor mutational burden (TMB) has been associated with greater efficacy of pembrolizumab in NSCLC (11). TMB typically translates into a higher neo-antigen load, and therefore a higher chance that an antigen capable of stimulating an immune reaction is expressed on the tumor cell surface and recognizable by a cytotoxic T-cell (Figure 1) $(11,12)$. TMB was recently confirmed in a randomized clinical study to be a powerful biomarker, independent of PD-L1 expression, in NSCLC patients treated with the single agent PD-1 inhibitor nivolumab, and also in studies combining the PD-1 and CTLA-4 inhibitors, nivolumab and ipilimumab $(6,13)$.

SCLC is an aggressive tumor, which represents about $10-15 \%$ of all lung cancers. Currently approved frontline therapies have limited efficacy for extensive stage SCLC (ES-SCLC): while initial therapy is usually very effective, remissions are usually short lived and relapses are almost universal. Median survival for patients with ES-SCLC 


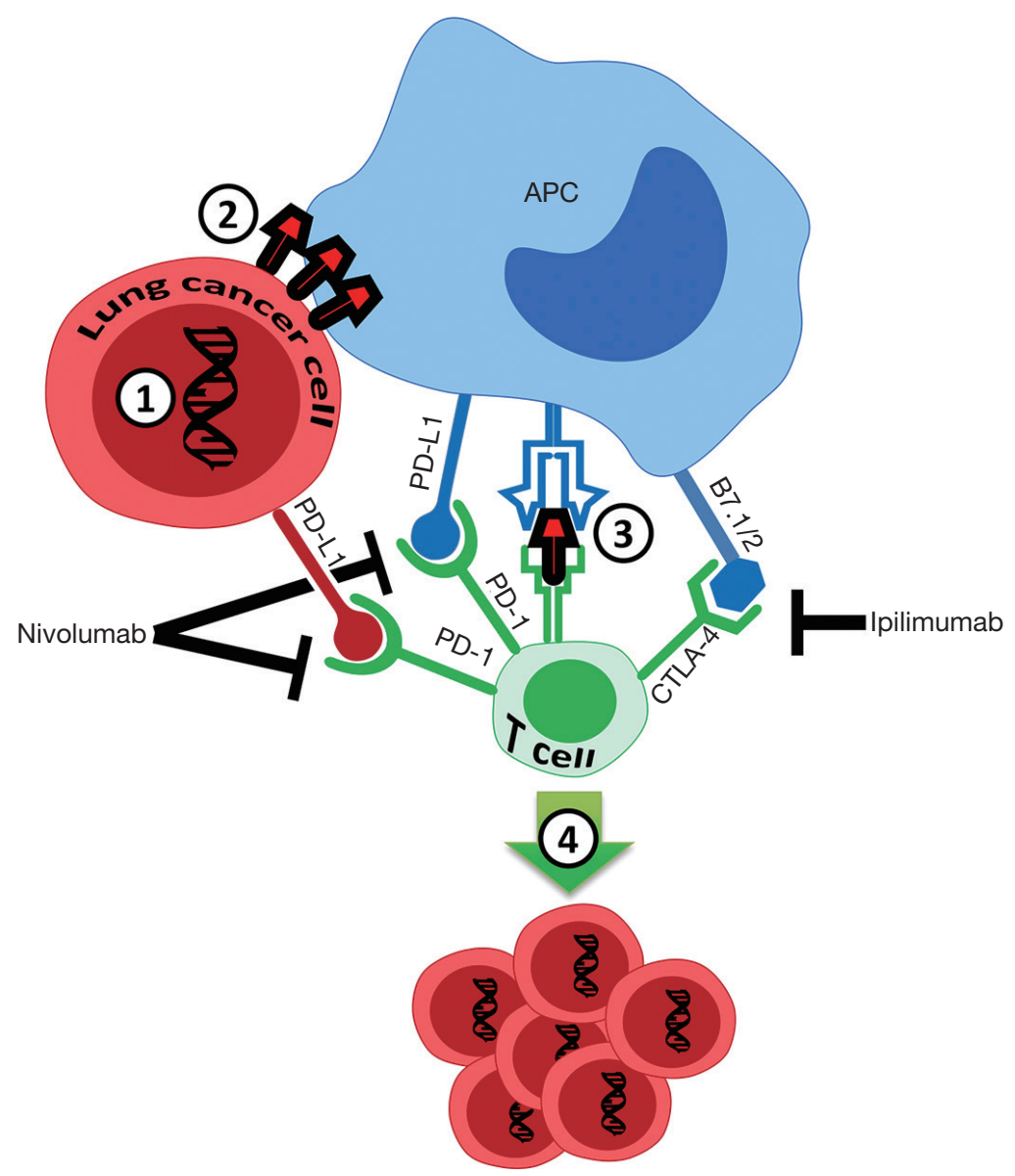

Figure 1 Small cell lung cancer (SCLC) cell mutational burden, and PD-1 and CTLA-4 inhibitor response. 1, mutations in SCLC cells, mostly related to smoking, generate neo-antigens; 2, neo-antigens are expressed on the cancer cell surface; 3, antigen presenting cells (APCs) recognize neo-antigens, and present them to CD8+ T-cells, inducing cytotoxic T-cell responses; 4, cytotoxic CD8+ T-cell activation occurs, resulting in robust neo-antigen-dependent tumor cell death. Combination of two checkpoint inhibitors is effective against subset of SCLCs with high TMB that reach a threshold for robust CD8+ cytotoxic T-cell activation. B7.1/CD80 and B7.2/CD86 are proteins expressed on APC that bind to CTLA-4 on cytotoxic CD8+ T cells.

treated with systemic chemotherapy is typically only about 12 months (14). Major unresolved issues have been lack of effective options following treatment failure, and strategies to lengthen remission before treatment failure. Traditionally, the relapse of ES-SCLC from the first line chemotherapy has been divided into two categories: refractory relapse, which occurs within a 60-90-day treatment-free interval (TFI) after the first-line chemotherapy, and sensitive relapse, which occurs after at least 60-90 days of TFI (15). In refractory relapse SCLC, topotecan, a topoisomerase 1 inhibitor, has a second-line median overall response rate (ORR) of $5 \%$, associated with a 1-year overall survival (OS) of $9 \%$, whereas for SCLC sensitive relapse, median ORR to second-line topotecan is $17 \%$, with 1 -year OS of $27 \%$ (16). In spite of this limited efficacy, topotecan remains the only FDA-approved standard therapy option for second line treatment of SCLC, according to NCCN guidelines (17).

Immunotherapy development for SCLC has lagged behind that for NSCLC, and the efficacy of immunotherapy in this disease appears to be lower than for NSCLC. The idea of adaptive immune resistance, where immunecheckpoint ligand such as PD-L1 is induced in tumors in response to an endogenous antitumor immune response suggests that PD-1 pathway blockade as a monotherapy will succeed only if antitumor immune responses pre-exist in the host, a cancer patient (1). Therefore, it is likely that 
SCLC patients that do not respond to immunotherapy with single agent checkpoint inhibitor have low PD-L1 expression and therefore lack pre-existing anti-tumor T-cell responses. Based on this idea, it has been proposed that a more effective approach for some tumor types would be the combination of PD- 1 and CTLA-4 inhibitors, in accord with the observation that combination nivolumab and ipilimumab have been FDA approved for metastatic melanoma in 2015, and for intermediate risk and high risk metastatic renal cell carcinoma and mismatch repair (MMR)-deficient colon cancer in 2018 (18-20). This combination therapy is now being actively studied across many other tumor types. The fact that nivolumab and ipilimumab have different mechanisms of action explains their synergistic effect: while nivolumab primarily blocks the tumor cell and cytotoxic CD8+ T-cell interaction via blocking an activated PD-L1 pathway, ipilimumab disrupts the $\mathrm{T}$ cell interaction with other APCs, such as dendritic cells, macrophages or B-cells (Figure 1).

An initial publication of a nonrandomized cohort of advanced SCLC patients treated with nivolumab, or nivolumab plus ipilimumab, showed 2-year OS of $14 \%$ and $26 \%$, respectively (8), which lead to a recommendation of the inclusion of nivolumab with or without ipilimumab treatment as part of the NCCN guidelines. This finding also contributed to a recent 2018 announcement by the FDA that the use of nivolumab for SCLC would receive priority review $(17,21)$. The role of $\mathrm{PD}-\mathrm{L} 1$ as a biomarker in SCLC has been controversial; initial studies found that SCLC has lower PD-L1 expression ( $\sim-30 \%$ of cells in tumor positive), and did not predict responses in SCLC compared to NSCLC. However, the recent Keynote-158 SCLC phase 2 study showed that PD-L1 could be detected in about $40 \%$ of SCLC, and was predictive of pembrolizumab clinical activity $(7,22,23)$.

In a key 2018 study by Hellmann et al. (24), a detailed biomarker analysis was performed on 211 (53\%) of 401 patients with ES-SCLC progressing after two or more lines of therapy, who had then been treated with nivolumab, or nivolumab plus ipilimumab, in the Checkmate 032 study. Sixty-nine $(27 \%)$ were TMB-high ( $\geq 248$ mutations per tumor) as determined by whole-exome sequencing (WES), while $73 \%$ had low or medium TMB. The WES results correlated well with in silico analysis of a smaller subset of FoundationOne 315 gene set, suggesting that FoundationOne CDx assay could be used for routine clinical testing of TMB. Strikingly, among the TMB-high patients the 1 -year OS was 35\% for patients treated with nivolumab, and $62 \%$ for patients treated with nivolumab and ipilimumab. These results were indeed highly promising among these heavily pretreated patients with an aggressive disease, and clearly numerically superior to 20-26\% OS in low/medium TMB patients that received nivolumab +/- ipilimumab. In contrast to TMB, PD-L1 was positive among $12 \%$ patients and was not predictive of responses.

Several conclusions can be made from the study. First of all, the results suggest that TMB is a robust predictive biomarker for checkpoint immunotherapy in metastatic SCLC, and the first reliable tissue-based biomarker for immune checkpoint blockade (ICB) in this disease. Second, SCLC patients, and especially the high-TMB population, which represents about $1 / 4$ of all SCLC, can clearly have long term benefit from nivolumab with or without ipilimumab therapy. The combination therapy shows robust synergy and looks promising and numerically superior to nivolumab with reported 1-year follow-up, and represents the first major breakthrough in this aggressive disease in decades.

Some unresolved issues remain before adoption of TMB as a routine clinical practice biomarker. Tissue acquisition is likely to be a problem for TMB assessment, as many patients with SCLC routinely receive only small cytology sample biopsies; therefore, a shift in this approach for patients with SCLC is needed to require core biopsies and perform routine TMB testing on majority of the patients. Future studies are needed using the FoundationOne CDx or similar panels or platforms, to determine if these yield results suitable for routine clinical practice. This would allow the replacement of WES with routine TMB. It will be important to clarify the TMB cutoff values specific to SCLC, to identify who will receive optimal benefit. Finally, it would be interesting to see an impact of TMB as predictive biomarker in other ongoing and other future planned studies in SCLC patients: a phase 1/2 combining nivolumab with or without ipilimumab with Rova-T DLL4 directed antibody drug conjugate (NCT03026166), a large phase 3 immunotherapy combination study which evaluates nivolumab with or without ipilimumab after completion of platinum chemotherapy, CheckMate 451 study (NCT02538666) and other studies combining various immunotherapy drugs with each other and with chemotherapy in SCLC. Future novel immunotherapy combination studies in SCLC contrasting response in specific TMB high $v s$. TMB low/medium patient subgroups are anticipated. 
Immunotherapy is improving the treatment of SCLC, and some patients are clearly benefiting from this class of therapeutic. To maximize treatment efficacy and to minimize severity of adverse events, establishing prognostic and response predictive markers is essential. Several factors can regulate immunotherapy responses, and the current study clearly shows that TMB is clearly a robust predictive biomarker. In contrast, more studies are needed to clarify the role of PD-L1 in SCLC, both in terms of prevalence of PD-L1 expression, and in evaluating its ability to predict responses. In spite of these advances, SCLC remains a tumor that is difficult to treat, with the majority of patients succumbing to this devastating disease. However, hopes are high that future studies using novel combinations and TMB as biomarker will improve outcomes, inducing long term remissions among SCLC patients, within the next few years.

\section{Acknowledgements}

The author thanks Galina Semenova, MD, PhD of Fox Chase Cancer Center for helping generate Figure 1.

Funding: The author is supported by the NIH R01 CA218802 grant, NIH R21 CA223394 grant, Fox Chase Cancer Center Clinical Protocol Development Award, the Russian Foundation for Basic Research Grant 18-44160004, and in part by the NCI Core Grant P30 CA006927 to Fox Chase Cancer Center.

\section{Footnote}

Conflicts of Interest: The author has served on advisory boards of Astra Zeneca, Boehringer Ingelheim, BristolMeyers Squibb, Caris Life Sciences, Novartis and Takeda.

\section{References}

1. Pardoll DM. The blockade of immune checkpoints in cancer immunotherapy. Nat Rev Cancer 2012;12:252-64.

2. Wolchok JD, Saenger Y. The mechanism of anti-CTLA-4 activity and the negative regulation of T-cell activation. Oncologist 2008;13 Suppl 4:2-9.

3. Bansal P, Osman D, Gan GN, et al. Recent Advances in Immunotherapy in Metastatic NSCLC. Front Oncol 2016;6:239.

4. Iwai Y, Hamanishi J, Chamoto K, et al. Cancer immunotherapies targeting the PD-1 signaling pathway. J Biomed Sci 2017;24:26.

5. Ribas A, Wolchok JD. Cancer immunotherapy using checkpoint blockade. Science 2018;359:1350-5.

6. Hellmann MD, Ciuleanu TE, Pluzanski A, et al. Nivolumab plus Ipilimumab in Lung Cancer with a High Tumor Mutational Burden. N Engl J Med 2018;378:2093-104.

7. Chung HC, Lopez-Martin JA, Kao SCH, et al. Phase 2 study of pembrolizumab in advanced small-cell lung cancer (SCLC): KEYNOTE-158. J Clin Oncol 2018;36: abstr 8506.

8. Hellmann MD, Ott PA, Zugazagoitia J, et al. Nivolumab (nivo) \pm ipilimumab (ipi) in advanced small-cell lung cancer (SCLC): First report of a randomized expansion cohort from CheckMate 032. J Clin Oncol 2017;35:8503.

9. Herbst RS, Baas P, Kim DW, et al. Pembrolizumab versus docetaxel for previously treated, PD-L1-positive, advanced non-small-cell lung cancer (KEYNOTE-010): a randomised controlled trial. Lancet 2016;387:1540-50.

10. Reck M, Rodriguez-Abreu D, Robinson AG, et al. Pembrolizumab versus Chemotherapy for PD-L1Positive Non-Small-Cell Lung Cancer. N Engl J Med 2016;375:1823-33.

11. Rizvi NA, Hellmann MD, Snyder A, et al. Cancer immunology. Mutational landscape determines sensitivity to PD-1 blockade in non-small cell lung cancer. Science 2015;348:124-8.

12. Forde PM, Chaft JE, Smith KN, et al. Neoadjuvant PD-1 Blockade in Resectable Lung Cancer. N Engl J Med 2018;378:1976-86.

13. Carbone DP, Reck M, Paz-Ares L, et al. First-Line Nivolumab in Stage IV or Recurrent Non-Small-Cell Lung Cancer. N Engl J Med 2017;376:2415-26.

14. Powell HA, Tata LJ, Baldwin DR, et al. Treatment decisions and survival for people with small-cell lung cancer. Br J Cancer 2014;110:908-15.

15. Ardizzoni A, Tiseo M, Boni L. Validation of standard definition of sensitive versus refractory relapsed small cell lung cancer: a pooled analysis of topotecan second-line trials. Eur J Cancer 2014;50:2211-8.

16. Horita N, Yamamoto M, Sato T, et al. Topotecan for Relapsed Small-cell Lung Cancer: Systematic Review and Meta-Analysis of 1347 Patients. Sci Rep 2015;5:15437.

17. NCCN Network. Clinical practice guidelines in oncology. Small cell lung cancer. Version 1. 2018. Available online: https://wwwnccnorg/store/login/ loginaspx?ReturnURL=https://wwwnccnorg/ professionals/physician_gls/pdf/sclcpdf

18. Larkin J, Chiarion-Sileni V, Gonzalez R, et al. Combined Nivolumab and Ipilimumab or Monotherapy in Untreated Melanoma. N Engl J Med 2015;373:23-34. 
19. Motzer RJ, Tannir NM, McDermott DF, et al. Nivolumab plus Ipilimumab versus Sunitinib in Advanced Renal-Cell Carcinoma. N Engl J Med 2018;378:1277-90.

20. Overman MJ, Lonardi S, Wong KYM, et al. Durable Clinical Benefit With Nivolumab Plus Ipilimumab in DNA Mismatch Repair-Deficient/Microsatellite Instability-High Metastatic Colorectal Cancer. J Clin Oncol 2018;36:773-9.

21. Broderick JM. FDA Grants Nivolumab Priority Review for SCLC. Onclive.com. Available online: https:// wwwonclivecom/web-exclusives/fda-grants-nivolumabpriority-review-for-sclc

Cite this article as: Boumber Y. Tumor mutational burden (TMB) as a biomarker of response to immunotherapy in small cell lung cancer. J Thorac Dis 2018;10(8):4689-4693. doi: 10.21037/jtd.2018.07.120
22. Antonia SJ, Lopez-Martin JA, Bendell J, et al. Nivolumab alone and nivolumab plus ipilimumab in recurrent smallcell lung cancer (CheckMate 032): a multicentre, openlabel, phase 1/2 trial. Lancet Oncol 2016;17:883-95.

23. Schultheis AM, Scheel AH, Ozretic L, et al. PD-L1 expression in small cell neuroendocrine carcinomas. Eur J Cancer 2015;51:421-6.

24. Hellmann MD, Callahan MK, Awad MM, et al. Tumor Mutational Burden and Efficacy of Nivolumab Monotherapy and in Combination with Ipilimumab in Small-Cell Lung Cancer. Cancer Cell 2018;33:853-61.e4. 